\title{
Development of Engineering Application for a Choice of Data Converters with Sigma Delta Architecture
}

\author{
Vasily N. Ashanin ${ }^{1}$, Boris V. Chuvykin ${ }^{2}$, Aleksey A. Korotkov ${ }^{1}$, Irina A. Sidorova ${ }^{2}$ \\ ${ }^{1}$ Department of electrical engineering, Penza State University, Penza, 440026, Russian \\ Federation \\ ${ }^{2}$ Department of information and measuring systems, Penza State University, Penza, 440026, \\ Russian Federation
}

Keywords: Sigma-delta modulator, sigma-delta ADC, sigma-delta architecture, data converters, decision support system, choice of the optimal decision, parameters of integrated microcircuits, database

\begin{abstract}
In article reasons and development of engineering application in the form of decision support system with the integrated database of sigma-delta data converters are presented. Authors carried out the analysis of the sigma-delta data converters' parameters. Sigma-delta data converters released by leaders' vendors in this branch of integrated microcircuits - Analog Devices, Texas Instruments/Burr-Brown, Maxim Integrated Products. The most significant of parameters allow to justify and to choose the electronic and component basis according to requirements of the development engineer are defined. Decision support system has two user's levels differing in professional preparation and levels of a task's specifications are provided: Beginner mode and Expert mode.
\end{abstract}

\section{Introduction}

The data converters (DC) with sigma-delta architecture ( $\Sigma \Delta$-architecture) possess unique metrological and exploitation properties: high linearity of a conversion function and noise immunity, low energy consumption. It explains their broad application in the industrial and research equipment, medical equipment, audio-and video equipment and in many other classes of devices for highprecision data processing. Thanks to possibility of their technical implementation completely on digital technology and achievements in the field of production of inexpensive digital integrated microcircuits, DC with $\Sigma \Delta$-architecture are used in "systems on a crystal" [1], [6], [14].

The main vendors of data converters are some foreign companies the largest of which are Texas Instruments/Burr-Brown [13], Analog Devices [11], Maxim Integrated Products [12]. The quantity of such chips continuously grows and totals as of the end of 2014 already about 250 names [2]. Domestic producers also joined process of release of integral DC with $\Sigma \Delta$-architecture, solving the problem of import substitution [7], [8]. The purpose of this paper is development of engineering application for purposeful choice of DC with $\Sigma \Delta$-architecture.

\section{Discussed Problem}

Distinctive feature of DC with $\Sigma \Delta$-architecture is the heterogeneity of representation of a signal in path of information conversion and, as a consequence, essential dependence of their metrological and exploitation properties on their structural implementation and functioning algorithm $[1,4,10]$. Creation of engineering application in the form of decision support system (DSS) allowing the developer of electronic equipment to realize a purposeful choice of DC with $\Sigma \Delta$-architecture is expedient.

The main difficulty of quick search satisfying according to characteristics of a chip in design process is absence of information in an explicit form of the architectural features and metrological characteristics defining effective number of bits, a modulator order, quantizer level, quantity of stages, a type of the digital filter, etc. Therefore authors systematized information on DC with $\Sigma \Delta$ - 
architecture of the main vendors, according to the classification signs given in papers of authors [1], [4].

In a basis of DSS's creation the task of development of the intuitive interface and support of filtering parameters of integral DC with $\Sigma \Delta$-architecture is set. DSS has the possibility of installation of priority and a choice of best values selection parameters

\section{Choice of the Parameters of DC with $\Sigma \Delta$-Architecture}

The nomenclature of the basic metrological, technical and exploitation parameters specified by venders in converters' DataSheets on reaches more than twenty. For making decision about significant parameters in the developing DSS by optimization of a choice of DC with $\Sigma \Delta$ architecture, authors carried out the analysis of integrated microcircuits characteristics of the leading firms. A row of general properties and parameters inherent in all to the let-out integral DC is defined.

Database (DB) of DC with $\Sigma \Delta$-architecture containing the complete information about the name and type of converters and the major parameters is created: "Accuracy", "High-speed performance", "Power", "Price", "Casing type", "Interface type", "Form of output signal", "Effective number of bits", "Vendor", "Integrator type", "Sampling rate", "Number of channels", "A modulator order", "Quantity of stages", "Quantizer level", "The type of filter", and also are specified "Year of release", the "Notes" and links to the "Page of documentation". The parameters which didn't enter this list are specified in the accompanying documentation to each chip (DataSheet).

The analysis of application of integral DC with $\Sigma \Delta$-architecture in difficult information measuring systems and devices for the priority directions of science and technique was held. As the main parameters are selected the parameters defining metrological and exploitation properties of the developed devices is offered. The following division of chips' characteristics on groups is offered:

High-speed performance: high (over 10M of samples per second), average (from 10k to 10M), small (to 10k), where $\mathrm{k}$ - a kilo (103), M - mega (106);

1) accuracy (ENOB - Effective Number of Bits): high (from 20 bits), average (16-20 bits), low (to 16 bits);

2) power consumption: low-power (to $100 \mathrm{~mW}$ ), powerful (over $100 \mathrm{~mW}$ );

3 ) the declared number of bits: high (from 20 bits), average (16-20 bits), low (to 16 bits);

4) interface type: serial, parallel, other (I2C, SPI, 2-Wire, Parallel CMOS, Parallel LVDS);

5) casing type: PDIP, CDIP, SOIC, TSSOP, VSSOP, SSOP, QSOP, MSOP, SOP, SOT, QFN, TQFN, VQFN, WQFN, LQFP, MQFP, TQFP, HTQFP, NFBGA, $\mu$ MAX, XCEPT, CFP, LFCSP, WLCSP, PLCC;

6) form of output signal: analog (current), digital;

7) price: expensive (over $\$ 50$ ), averages ( $\$ 10-50)$, inexpensive (to $\$ 10$ ).

\section{DSS Operation Modes}

For the purpose of extension a circle of engineering application users two operation modes are realized: Beginner mode and Expert mode. The Beginner mode is intended for the specialistdeveloper from adjacent areas of science and technique. The developers have the general principles of creation and implementation of measuring devices and systems. Using DSS in the Beginner mode has information and fact-finding character and doesn't allow to realize action for editing a DB. This mode assumes a selection of converter type on values of parameters without possibility of exposure a priority of search.

Functioning of DSS in the Beginner mode assumes the following items:

1) Filtering of chips from the type DC with $\Sigma \Delta$-architecture: specialized (Front-End), singlechannel sigma-delta of ADC, multi-channel sigma-delta of ADC, sigma-delta modulators;

2) Chips are filtered on the parameter values selected by user;

3) The table with selected DC with $\Sigma \Delta$-architecture satisfying the parameter values given user is result of working DSS. 
For the professional design engineers solving complex problems of processing of analog-todigital information it is offered to use the Expert mode in the help. Feature of operation of DSS in this mode is selection of chips in the given parameters with arrangement of priorities, and also possibility to users of independent updating of a DB.

Functioning of DSS in the Expert mode repeats the first and the second points of functioning algorithm of DSS in the Beginner mode, further application works as follows:

1) Exposure sequence of search the priorities in the selected values of parameters which level of importance is put down on a ball scale from 1 to $8(1-$ the most important, 8 - the least important). By default all values of priorities are set as "1".

2) Further the system in that sequence in which there were preset values of priorities, makes filtering values of DB since the higher priority on the decreasing. Filtering is realized by a choice of values of the corresponding parameters on a mutually exclusive basis in favor of chips with higher priority.

The table with selected by DC with $\Sigma \Delta$-architecture, satisfying user's parameter values and their priorities, with possibility of sorting according to increase/decrease in alphabetical order is result of operation of DSS.

Application is written in language $\mathrm{C}++$ with use of the Qt-5 library having a wide functionality and the convenient user interface in Russian (Fig. 1).

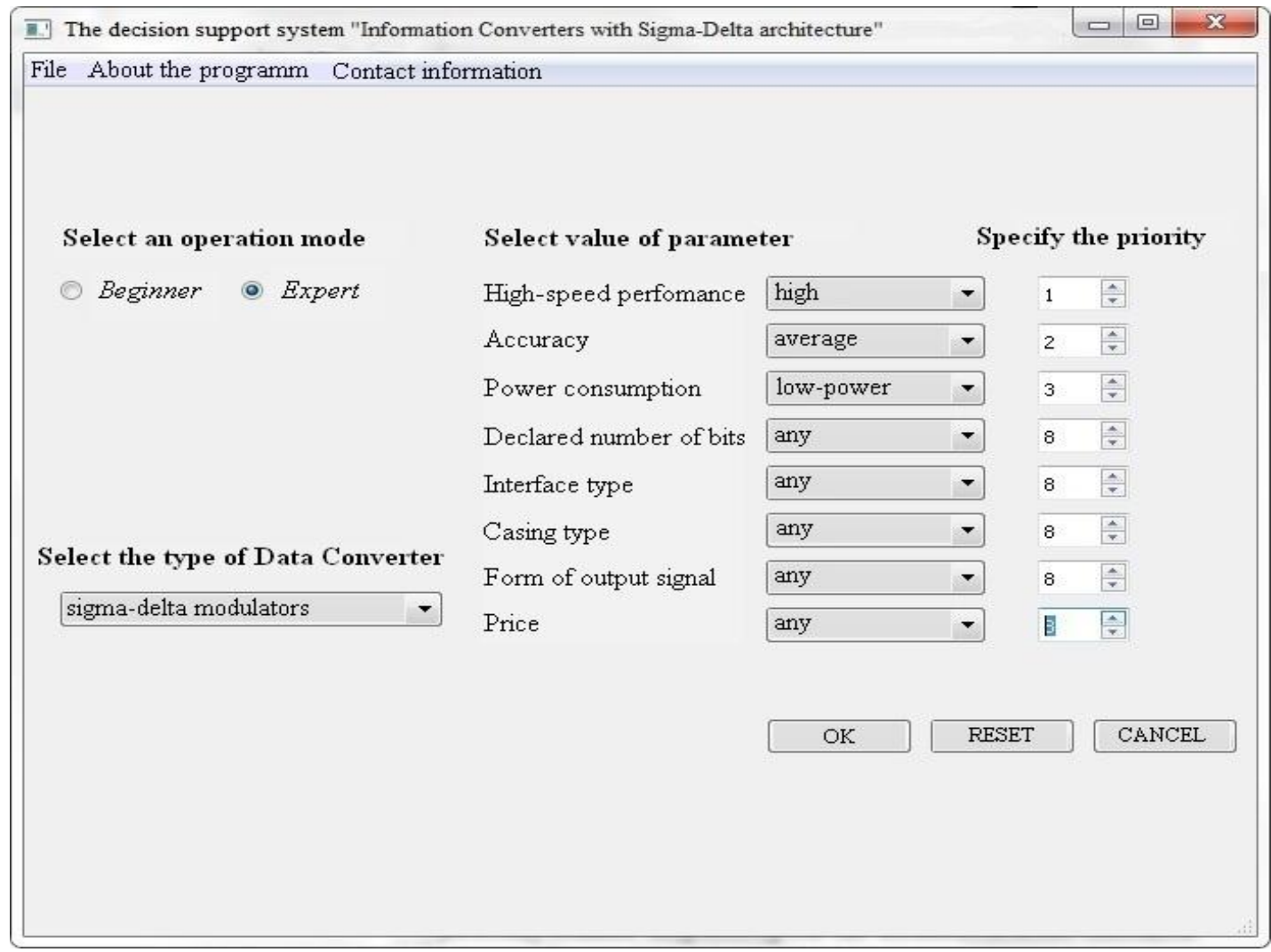

Fig. 1. The system interface in Expert mode.

\section{Conclusions}

The paper presents an innovative DSS for selecting DC with $\Sigma \Delta$-architecture. The offered DSS is unique on the functionality among development of open access for this specialized subject area.

Therefore developed DSS can be used not only as an engineering application for solving the problem of choosing the chip, but also as a reference manual to set the optimal parameters of the device being modeled in CAD. 
Designed DSS for selecting DC with $\Sigma \Delta$ architecture are registered as a "computer program" in the Federal service for intellectual property $[5,9]$ and is a local application that can be installed on any computer suitable according to system requirements.

Authors hope that application will be demanded due to growth of level of demand on DC with $\Sigma \Delta$-architecture both abroad and in Russia.

\section{References}

[1] Ashanin, V. N., Chuvykin, B. V., Shakhov, E. K. $\Sigma \Delta$ ADC: Basic theory and design. Monograph. Information and publishing centre of Penza State University, 2009, 188 p.

[2] Ashanin, V. N., Chuvykin, B. V., Korotkov, A. A., Sidorova, I. A. Analysis of condition and trends in production of integral data converters of $\Sigma \Delta$-architecture. Proceedings of the universities: The Volga Region. Technical Science, no. 1 (29), 2014, pp. 26-35.

[3] Ashanin, V. N. Classification of information transducers in continuous-discrete system of the heterogeneous structure. Proceedings of the universities: The Volga Region. Technical Science, no. 3, 2011, pp. 98-104.

[4] Ashanin, V. N. Analysis and synthesis theoretical problems of the measurement on the heterogeneous structure. Sensors and Systems, no. 7, 2011, pp. 2-7.

[5] Database "Information Converters with Sigma-Delta architecture". Ashanin, V. N., Chuvykin, B. V., Korotkov, A. A., Sidorova, I. A. The Certificate on official registration of the database. No. $2014621524,2014$.

[6] Kester U. Analog-to-digital conversion. Technosphere, 2007, 1015 p.

[7]Scientific and production center «ELVIS». Available at: http://www.multicore.ru/download.php?file=mc/data_sheets/ELVEES_catalogue.pdf

[8] Open Joint Company «NIIET». Available at: http://www.niiet.ru/chips/dais?id=166

[9] The decision support system "Information Converters with Sigma-Delta architecture". Ashanin, V. N., Chuvykin, B. V., Korotkov, A. A., Sidorova, I. A. The Certificate on official registration of the computer program. No 2014660777, 2014.

[10] Chuvykin, B. V., Dolgova, I. A., Sidorova, I. A. Technique of definition the low-frequency periodic vibrations in single-bit signals of sigma-delta modulators. Caspian Journal: Management and High Technology, no. 2 (26), 2014, pp. 174-181.

[11]Analog to digital converters [Electronic resource]. Available at: http://www.analog.com/en/analog-to-digital-converters/ad-converters/products/index.html

[12]Precision Sigma-Delta ADCs. [Electronic resource]. Available at: http://www.maximintegrated.com/products/data_converters/adcs/precision-sd-adc.cfm

[13]Products for Analog to Digital Converter. [Electronic resource]. Available at: http://www.ti.com/lsds/ti/data-converters/analog-to-digital-converter-products.page

[14] Schreier, R., Temes, G. C. Understanding Delta-Sigma Data Converters. IEEE Press. 2008, 446 p. 